\title{
Perioperative chemotherapy in the treatment of osteosarcoma: a 26-year single institution review
}

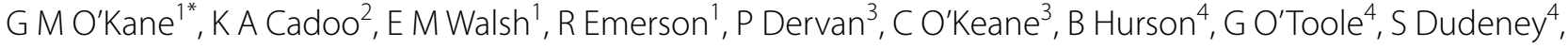 \\ E Kavanagh $^{5}$, S Eustace ${ }^{5}$ and D N Carney ${ }^{1}$
}

\begin{abstract}
Background: Chemotherapy in the multimodality treatment of osteosarcoma has improved survival. Reported outcomes on adult patients are limited. Poor necrosis rates post neoadjuvant chemotherapy (NAC) is considered an adverse prognostic factor and attempts have been made to improve survival in this group.
\end{abstract}

Patients and methods: Adult and young adult patients diagnosed with osteosarcoma between January 1986 and August 2012 were retrospectively reviewed. Patients identified were stratified according to stage (localised or metastatic) and age ( $\leq 40$ and $>40$ years). Event free survival (EFS) and overall survival (OS) outcomes were determined. In patients with localised disease $\leq 40$ years, survival was assessed according to necrosis rates post NAC ( $<90$ and $\geq 90 \%)$. NAC consisted of two cycles of methotrexate alternating with doxorubicin/cisplatin (MAP) followed by definitive surgery. Those with $\geq 90 \%$ tumour necrosis continued on MAP. Patients with $<90 \%$ necrosis received ifosfamide and etoposide (IE) post operatively.

Results: A total of 108 patients were reviewed and 97 were included. Median age was 23 years (range 16-75) and $70 \%$ of patients were male. Five year EFS and OS across all groups was $57 \%$ and $63 \%$ respectively. Of the patients with localised disease $(N=81)$, 5-year overall survival (OS), with a median follow up of 7 years $(2-26)$ was $70 \%(p<0.0001)$. Patients aged 16-40 ( $N=68)$ with localised osteosarcoma had a significantly improved 5-year OS $(74 \%)$ compared to those $>40$ years $(N=13)(42 \%)(p=0.004)$. Of the 68 patients with localised osteosarcoma $\leq 40$ years, 62 were evaluated according to necrosis rates post MAP. In 33 patients who achieved $\geq 90 \%$ necrosis and continued MAP, 5-year OS was $82 \%$. In 29 patients who had $<90 \%$ tumour necrosis and received adjuvant IE, 5 -year OS was $68 \%(p=0.15)$. Multivariate analysis confirmed age and stage as prognostic factors but not poor necrosis rates in our treated population.

Conclusions: Long-term survival outcomes in a predominantly adult Irish population are similar to large reported trials. Age and stage at diagnosis are prognostic. Postoperative ifosfamide/etoposide alone in patients with poor necrosis rates is a feasible regimen, but its role in the adjuvant setting remains uncertain.

Keywords: Adult osteosarcoma, Neoadjuvant chemotherapy, Poor necrosis rates, MAP, Ifosfamide/etoposide

\section{Background}

Osteosarcoma is a rare tumour type occurring with an incidence of $0.2-3 / 1,00,000$ per year in Europe [1]. Despite this, it is the most common primary tumour of

\footnotetext{
*Correspondence: okaneg@tcd.ie

1 Department of Medical Oncology, Mater Misericordiae University

Hospital, Eccles Street, Dublin 7, Ireland

Full list of author information is available at the end of the article
}

bone (excluding multiple myeloma), which occurs with a bimodal age distribution. The first peak, in adolescents and young adults, possibly coincides with the pubertal growth spurt. The second peak in patients over 65 years may result from the development of osteosarcoma as a secondary malignancy $[2,3]$. The metaphyses of long bones are the most commonly affected site [3] with more than $50 \%$ of occurrences adjacent to the knee joint [4]. 
Prior to the 1970s surgery alone cured $<20 \%$ of patients; the majority of deaths resulted from the rapid development of lung metastases [5]. Since the incorporation of chemotherapy, multimodality treatment has improved survival to approximately $70 \%$ at 5 years with little improvement in the last two decades $[4,6,7]$. Trials demonstrating this survival have largely included paediatric patients and outcomes in adult patients alone are limited.

Where feasible, the standard of care for patients with localised extremity osteosarcoma involves NAC followed by definitive surgical resection and adjuvant chemotherapy [8]. Although NAC versus immediate surgery and adjuvant chemotherapy has not been shown to improve OS [6], it does allow for optimal surgical planning [9] and avoids the need for amputation in most patients. Chemotherapy protocols have generally incorporated four active agents: Methotrexate with leucovorin rescue, doxorubicin, cisplatin and ifosfamide [10]. The most appropriate combination of these drugs however, continues to be debated [8].

NAC also provides prognostic information on tumour necrosis rates, which has been used to tailor postoperative treatment. Based on the Huvos grading system, patients with $\geq 90 \%$ necrosis are considered good responders and those with $<90 \%$ necrosis as poor responders [11]. Large trials have demonstrated inferior 5 -year survival rates in patients with a poor response versus those with a good response $(45-56 \%$ versus $71-80 \%$ respectively) $[4,12-14]$. Attempts in the past have failed to improve survival by modifying adjuvant treatment in poor responders and a recent meta-analysis found no benefit in switching or intensifying drugs $[7,15,16]$. Ifosfamide/etoposide (IE) has been shown to have efficacy in the metastatic setting [17] and is considered safe when added to MAP [18]. MAPIE formed the adjuvant arm of poor responders in the EURAMOS- 1 trial and provisional results reveal no improvement in survival [19]. At our institution patients with poor necrosis rates have been receiving postoperative IE alone since 1986.

In this article we review the outcomes of a predominantly adult population over a 26-year period and assess the impact of switching chemotherapy to IE in patients with $<90 \%$ necrosis rates post neoadjuvant MAP.

\section{Patients and methods}

\section{Patient population}

A retrospective review was undertaken of patients with a diagnosis of osteosarcoma seen by the oncology department at the Mater Misericordiae University Hospital from January 1986 to August 2012. This hospital has been a tertiary referral centre for the systemic treatment of adult osteosarcoma, collaborating with Cappagh
Hospital, the national orthopaedic hospital, where the majority of bone tumour surgeries are performed. All pathology was reviewed at our institution. During the period analysed, three orthopaedic surgeons performed $98 \%$ of the surgeries and the same oncologist delivered systemic treatment in all patients.

Patient details were obtained from the pathology department database and corroborated with the hospital information system search for patients with a diagnosis of osteosarcoma. Medical records of the patients collected were then appraised. Data was obtained on all patients with a histological diagnosis of high-grade osteosarcoma. We included patients with extremity or truncal high-grade osteosarcoma who received systemic treatment. Patients with craniofacial osteosarcoma, which is considered less aggressive, and those who did not receive chemotherapy were excluded. Patients were stratified according to stage: localised and metastatic disease at presentation and then further classified by age: $\leq 40$ and $>40$ years. Those patients with localised disease $\leq 40$ years, who received NAC were subsequently assessed according to necrosis rates based on the Huvos grading system [11]: poor responders $(<90 \%$ necrosis, grade I-II) and good responders $(\geq 90 \%$ necrosis, grade III-IV).

\section{Staging and treatment details}

Staging was performed using a plain film, radionuclide bone scan and/or magnetic resonance imaging (MRI) of the affected limb. Computed tomography (CT) thorax, abdomen and pelvis (TAP) and Chest X-Ray (CXR) completed radiological staging. More recently FDG PET/CT has been incorporated into routine work up. Full blood count and biochemical analyses were also measured at diagnosis and throughout treatment. The intended operating surgeon performed diagnostic biopsies where possible. All patients were discussed at our institutional multidisciplinary team meeting (MDT) at diagnosis, post $\mathrm{NAC}$ and post resection.

The systemic treatment of resectable osteosarcoma at our institution consists of two cycles of high dose methotrexate $8 \mathrm{~g} / \mathrm{m}^{2}$ alternating with doxorubicin $25 \mathrm{mg} / \mathrm{m}^{2}$ days $1-3$ and cisplatin $100 \mathrm{mg} / \mathrm{m}^{2}$ on day 1 (MAP), followed by definitive surgery and adjuvant chemotherapy (Additional file 1: Table S1). Patients with tumours which upon histological review demonstrate $\geq 90 \%$ necrosis continue MAP for a further two alternating cycles while those who have $<90 \%$ necrosis rates switch to ifosfamide $1,800 \mathrm{mg} / \mathrm{m}^{2}$ with mesna $1,800 \mathrm{mg} / \mathrm{m}^{2}$ days $1-5$ and etoposide $100 \mathrm{mg} / \mathrm{m}^{2}$ days $1-5$ for a total of four cycles (Additional file 1: Table S1). Dosing and scheduling of chemotherapy administered was confirmed with the pharmacy department. 
Patients with metastases at diagnosis who were considered resectable were treated with the same protocol and at surgery had resection of the primary lesion and metastases where applicable.

Close surveillance was initiated upon completion of adjuvant treatment. Patients were seen 3 monthly for the first 2 years with a CXR; a CT Thorax was performed every 6 months. Thereafter patients were reviewed 4-6 monthly with a CXR until year 5 at which point annual follow-up was commenced.

\section{Data collection}

Demographic and clinical details were extracted from the patient's medical records. Information on histology was obtained from the laboratory report system. Data collected included age, sex, stage at diagnosis, tumour site, type of operation, percentage necrosis, chemotherapy regimen used and number of cycles administered. Relapse date and site, together with survival and follow up information was also recorded.

\section{Statistical analysis}

Demographic details and treatment variables are reported by descriptive statistics. EFS and OS were computed from time of tissue biopsy until first recurrence or death respectively. Time of cut off for analyses was July 31st 2014. The Kaplan-Meier survival analysis method and the log rank test were used to estimate survival probability and compare survival within stratified groups. The Cox Regression model was used to assess the prognostic impact of age, stage and necrosis rates on survival. Analyses were conducted using SPSS 20.0

\section{Results}

\section{Patient characteristics}

Between January 1st 1986 and July 31st 2012108 adult patients with a diagnosis of osteosarcoma were reviewed by medical oncology. Eight patients who did not receive chemotherapy due to poor performance status (median age 73 years, range $46-81$ ) and three patients with craniofacial disease were excluded. A total of 97 patients were included. Detailed baseline patient demographics and tumour characteristics are available in Additional file 2: Table S2. Specific high-grade subtypes were incompletely reported during the time period and not available for analysis. Five patients were under 18 years (16-17 years) with the majority $(95 \%) \geq 18$ years. Median age overall was 23 years (16-73). Seventy percent of patients were male. The most common site of tumour was the femur $(54 \%)$ followed by the tibia $(20 \%)$. Of the total patients included $(N=97) 81(84 \%)$ had localised disease and $16(16 \%)$ had metastatic osteosarcoma at diagnosis. In patients with localised disease, 68/81 (84\%) were $\leq 40$ years $(16-40)$ and in patients with metastatic disease at diagnosis, 11/16 (68\%) were $\leq 40$ years (17-40). Follow-up for all patients ranged from 2 to 28.6 years with a median of 7 years.

\section{Surgical treatment}

Limb-salvage surgery was performed in $70 \%$ of patients with disease of the extremities $(N=90)$. Of these 90 patients 77 had localised disease and 13 metastatic disease. Limb-salvage was possible in $77 \%$ of patients with localised disease and was performed in only $31 \%$ of patients with metastatic disease (4/13) (Additional file 2: Table S2). Notably the period of this review from 1986 to 2012 has seen changes in approaches to the surgical management of extremity osteosarcoma with the increasing use of limb-salvage techniques in recent years [20,21].

\section{Chemotherapy}

In patients with localised disease $(N=81), 618$ of planned 648 chemotherapy cycles were completed (95\%). Eight patients (10\%) did not complete the intended cycles of treatment. Four patients $\geq 40$ years with localised disease, developed grade 3-4 toxicities, as did three patients $\leq 40$ years. One further patient $\leq 40$ years refused two final cycles of adjuvant treatment. Of the 16 patients with metastatic disease 11 (70\%) completed intended first line treatment.

\section{Survival outcomes}

The 5-year EFS and OS across all groups was 57 and $63 \%$ respectively. Stage at diagnosis was prognostic. Patients with localised disease $(N=81)$ had a significantly improved 5-year OS compared to those with metastatic disease at diagnosis $(N=16), 70$ versus $25 \%$ $p<0.0001$ (all age groups) (Figure 1a). EFS was 65 versus $13 \% p<0.0001$ (Figure 1b). Despite unbalanced numbers in each group age was also prognostic. EFS in localised disease $\leq 40$ years $(N=68)$ was $70 \%$ compared to $30 \%$ in those $>40$ years $(N=13)(p=0.009)$. Corresponding 5 -year OS was $74 \%$ compared to $42 \%$. ( $p=0.004$ ) (Figure $2 \mathrm{a}, \mathrm{b}$ ). Seven of the 13 patients $>40$ years (median age 54 years, range 44-74) with localised disease have died. Of these four were unable to complete chemotherapy due to toxicity. Multivariate analysis using the Cox Regression model confirmed the prognostic impact of age and stage for $\operatorname{EFS}(p=0.001$ and $p=0.001$ respectively) and for $\operatorname{OS}(p=0.002$ and $p=0.001$ respectively).

\section{Relapsed disease in patients with localised osteosarcoma at diagnosis}

Of the 81 patients with localised disease, 32 (40\%) experienced recurrences. This included seven patients $>40$ years ( $54 \%$ of all patients $>40$ years). The lung was site 

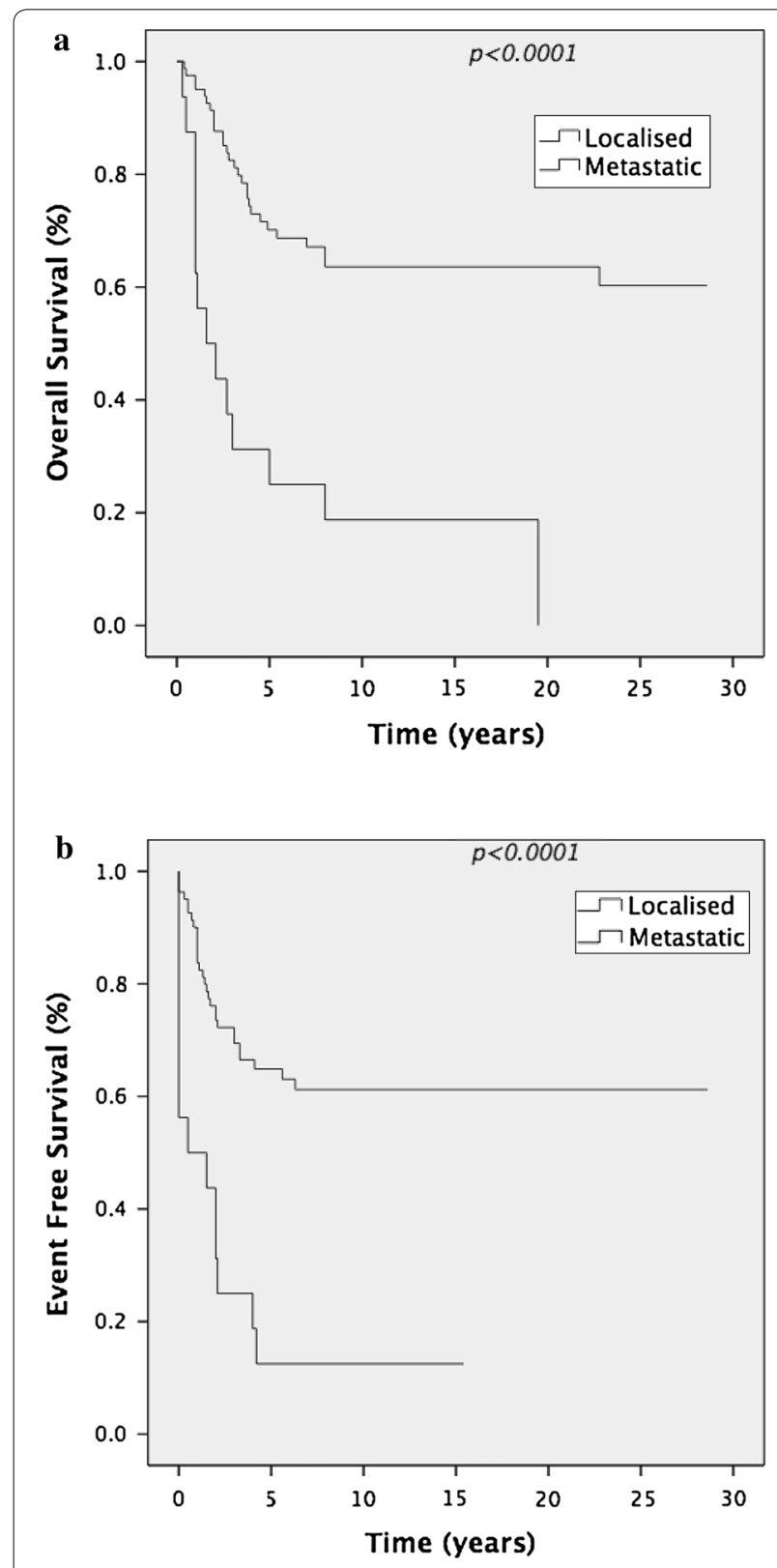

Figure 1 a Overall survival in patients with localised disease versus metastatic disease. $\mathbf{b}$ Event free survival in patients with localised disease versus metastatic disease.

of first recurrence in $22 / 32(69 \%)$ patients, with locally recurrent disease in nine patients. Three of these nine patients developed lung metastases within 1 year and one patient developed lung metastases 4 years later. One patient developed brain metastases as first documented recurrence but was also found to have lung metastases post complete imaging. Of the 32 patients who recurred, 11 patients $(9 \leq 40$ years and $2>40$ years) are alive and disease free with a median follow-up of 10.3 years (range
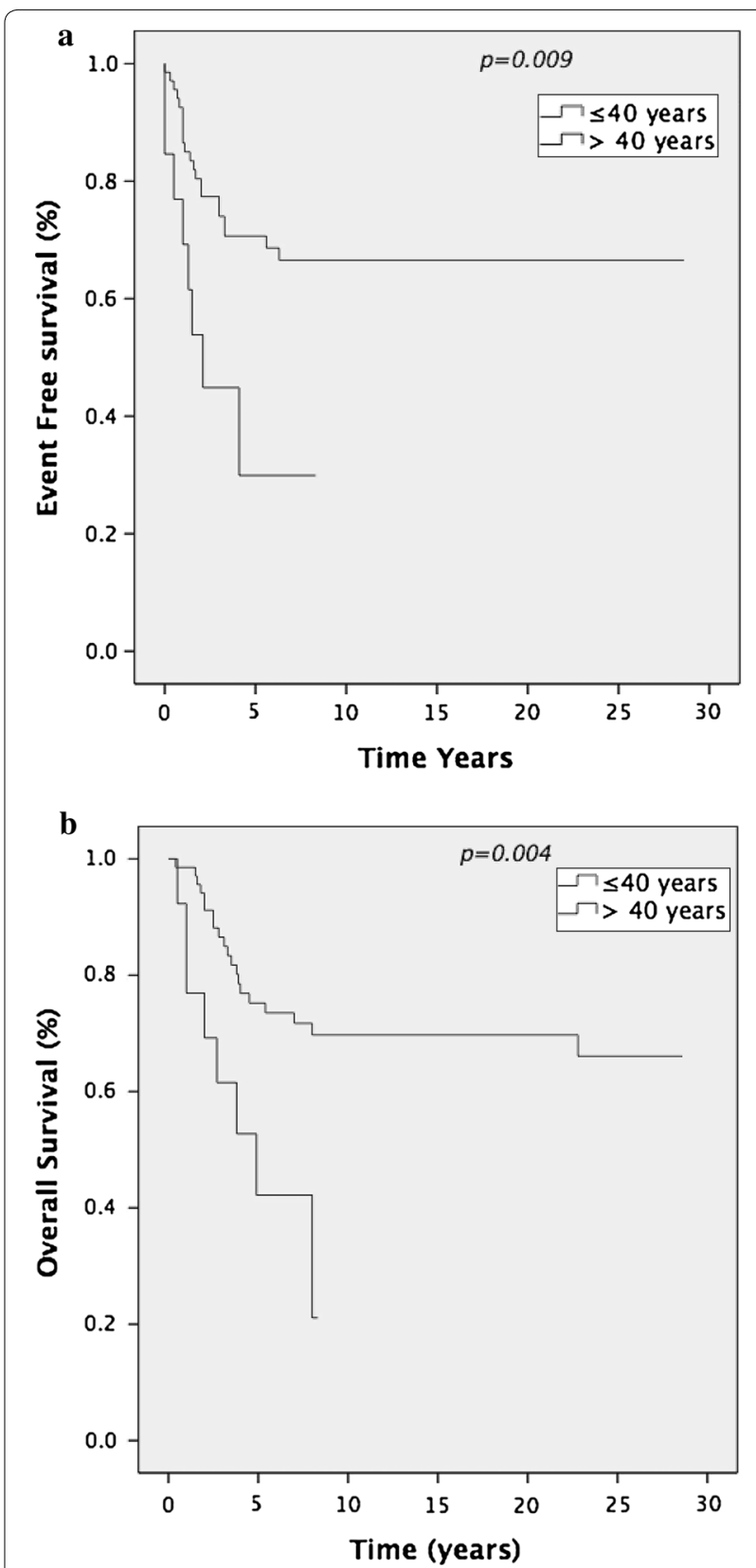

Figure 2 a EFS and $\mathbf{b}$ OS in localised disease according to age.

4.8-27.1). Four of these 11 patients developed local recurrences and were treated with surgery including one amputation; the remaining seven patients had thoracotomies for lung metastases (median number of thoracotomies 1; range 1-3).

\section{Impact of switching chemotherapy in patients with poor necrosis (localised $\leq 40$ years)}

Of the 68 patients with localised disease $\leq 40$ years, 62 were eligible for assessment of survival according to 
necrosis rates. The remaining six patients had upfront surgery without NAC and were thus excluded. Five of these six patients were initially thought to have parosteal osteosarcoma and one patient a chondrosarcoma. Pathological review of tumour specimens post resection revealed high-grade osteosarcoma. These six patients all received eight cycles of chemotherapy in the adjuvant setting and five are still alive with a median follow up of 3.8 years $(2.2-14.4)$.

The 62 patients included all had initial biopsies and post NAC histology reviewed at our institution. Median follow-up time in this group was 9.4 years (range 2-28.6). 33 patients demonstrated $\geq 90 \%$ necrosis post MAP and 29 patients $<90 \%$ necrosis. There was no significant difference in 5 year-EFS in good responders $(81 \%)$ and in poor responders $(64 \%)(p=0.18)$ (Figure 3a). Five year OS was $82 \%$ in those with $\geq 90 \%$ necrosis versus $68 \%$ in those with $<90 \%$ necrosis; this result was again not statistically significant $p=0.15$ (Figure $3 \mathrm{~b}$ ). When included in multivariate analysis, necrosis rates post NAC was not prognostic for EFS $(p=0.9)$ or OS $(p=0.62)$ in our cohort (Additional file 3: Table S3).

\section{Outcomes in patients with metastatic disease at diagnosis}

Sixteen patients (16\%) had evidence of metastases at diagnosis and 5-year OS was $25 \%$. Lung metastases were present in 13 patients and 3 patients had multifocal disease. Of the 13 patients with lung metastases 7 had thoracotomies, 2 at primary resection and 5 at recurrence. The median number of thoracotomies was 1 (range 1-4). Two patients with metastases at presentation, 1 with multifocal disease and 1 with lung metastases are still alive 8.3 and 15.4 years later.

\section{Discussion}

This is a retrospective study of predominantly adult osteosarcoma patients treated systemically at a single institution over 26 years. We have established that long term overall survival outcomes in this Irish population are comparable to previously reported large clinical trials. This study has also demonstrated the feasibility of IE postoperatively in patients with a poor response to neoadjuvant MAP.

Adult patients are generally underrepresented in osteosarcoma trials despite approximately half of patient diagnoses occurring in those over 20 years. [22] The median age in our study was 23 years with $95 \%$ of patients $\geq 18$ years and $70 \%$ of patients included were male. The male preponderance in osteosarcoma is well documented and although the proportion is slightly higher than reported ratios $[3,23]$ this may be explained by the older age of the patients included, as peak incidence in the female population occurs earlier [23].
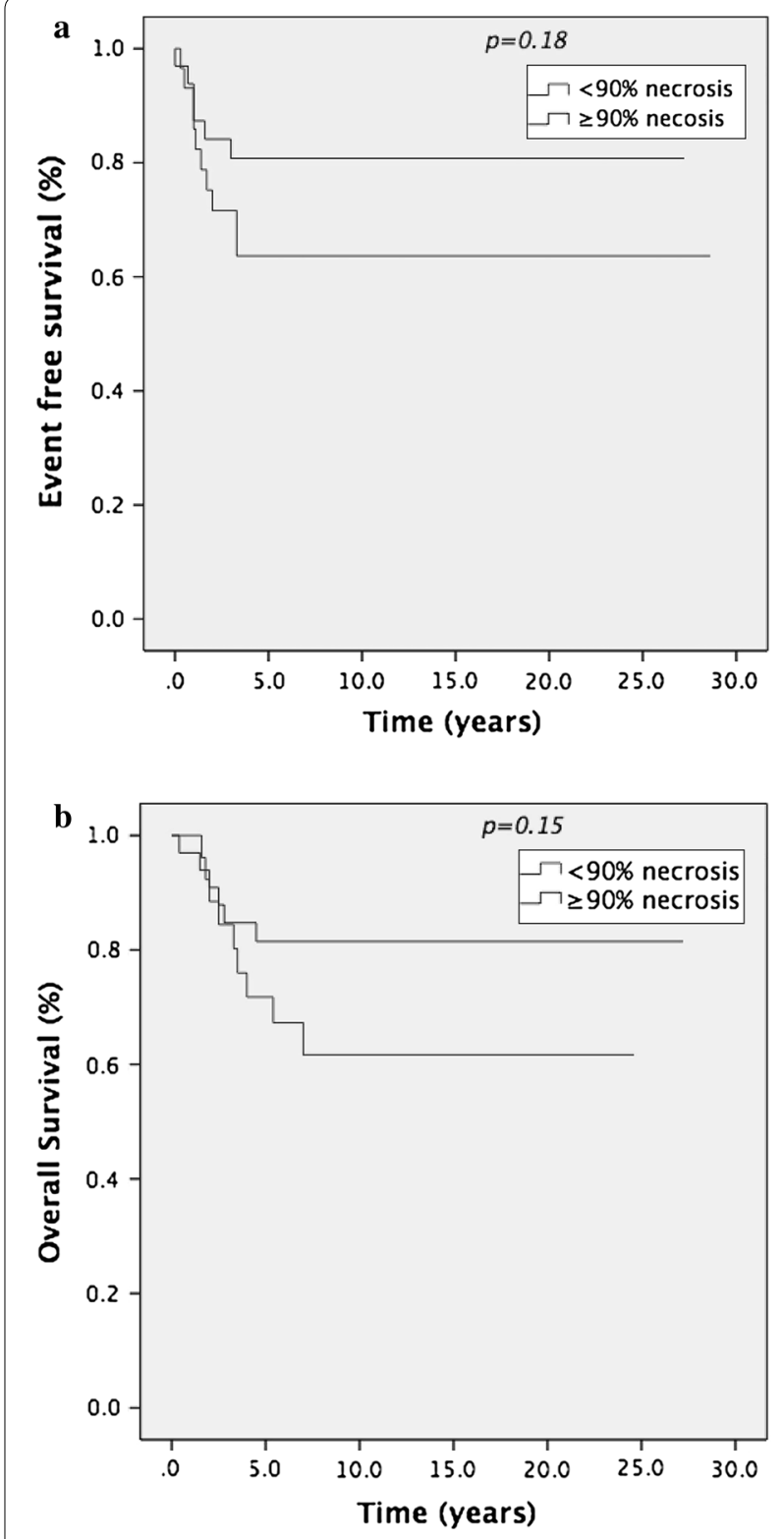

Figure 3 a EFS and $\mathbf{b} O S$ in patients with localised disease $<40$ years according to necrosis rates.

Inferior outcomes have been reported in patients over $40[24,25]$ and over 65 years [26]. A meta-analysis reported by Harting et al. investigating age as a prognostic factor in osteosarcoma, revealed that patients aged 21-40 $(N=110)$ had the same outcomes as that of the paediatric population with inferior survival in those $>40$ years $(N=56)$ [27]. This study acknowledges however that age alone is unlikely to contribute to inferior outcomes, but rather site, size and tumour necrosis rates in this group. In our study 5-year OS for all 97 patients included was $64 \%$, improving to $74 \%$ when selecting 
patients aged $16-40$ years with localised disease $(N=68)$. A small number of patients $(N=18)$ were $>40$ years at diagnosis. Of these, patients with localised disease at presentation $(N=13)$ had a significantly inferior survival at 5 years $(45 \%)$ compared to patients $\leq 40$ years. Seven of thirteen patients died and in four of these less than $50 \%$ of chemotherapy cycles were completed as a result of toxicity. The remaining three patients all had tumours $>8 \mathrm{~cm}$, one of whom had positive surgical margins; all three demonstrated poor necrosis rates post NAC. These factors are likely to have impacted on survival, however numbers are too small to make further conclusions. One of the largest studies in patients over 40 years reported by Grimer et al. [25] describes 238 patients with non metastatic osteosarcoma and reports a 5-year survival of $46 \%$. Other studies report superior survival rates of $55-62 \%$ at 5 years $[4,28]$. It is therefore likely that other patient and tumour characteristics contribute to outcomes.

Less than $20 \%$ of patients with osteosarcoma will present with de novo metastatic disease [29]. In our cohort $16 / 97$ (16\%) patients had metastatic disease at diagnosis. The 5 -year OS of $25 \%$ in this group is consistent with previous studies, which highlight number and resectability of metastases as prognostic in those with de novo metastatic disease $[29,30]$. Of patients with localised disease at diagnosis who recurred in our study, 11/32 (34\%) were salvaged with surgery. Seven of these patients were rendered disease free post thoracotomies. The aggressive surgical management of recurrent osteosarcoma with complete clearance is necessary for long-term survival and is well described [31-33]. Duration of relapse free interval and number of lesions are considered prognostic factors [31].

When evaluating the impact of poor necrosis rates on survival we were able to include 62 patients with localised disease $\leq 40$ years who received NAC. Responsiveness to NAC has been established as a predictor of survival [4, 12-14, 34].

The COSS, EOI and IOR groups have reported 5-year survival rates of $55.5,45$ and $56 \%$ respectively for poor responders with localised osteosarcoma [4, 12, 35]. Multiple studies have attempted to improve outcomes in this poor prognostic group by switching drugs or intensifying treatment, however a meta-analysis published by Anninga et al. [16] showed no benefit to either method.

MAP as a neoadjuvant regimen has been used since the 1980s and is considered standard of care in the United States [36]. This combination formed the backbone of the international multi-group EURAMOS-1 trial [37], the largest trial conducted in osteosarcoma. Survival outcomes in osteosarcoma have plateaued in recent decades and this group attempted to improve outcomes in both good and poor responders. The addition of IFN alpha- $2 b$ to MAP in good responders has recently been reported and failed to enhance survival [38]. Patients with poor necrosis rates were randomized to remain on MAP postoperatively or switch to MAP and IE.

The role of ifosfamide in osteosarcoma has been controversial. It has been shown to be active in the metastatic setting $[17,39]$ and has shown improvements in recurrence free survival and overall survival in patients at first recurrence [32]. The use of ifosfamide as a fourth drug in the neoadjuvant setting however does not improve histologic response and increases hematological toxicity [40]. When IE is added to MAP in poor responders post operatively results have differed. The IOR group have found favorable outcomes with use of ifosfamide [41] however the Scandinavian Sarcoma Group (SSG) failed to show a benefit of the addition of IE to MAP adjuvantly in poor responders [42]. Early results from EURAMOS-1 have also failed to show a benefit of additional IE to MAP in poor necrosis patients. It should be noted that fewer patients in this group received the intended doses and more toxicities were experienced [19]. In our patients survival at 5 years was $82 \%$ in good responders versus $68 \%$ in those treated with IE alone postoperatively. This difference was not statistically significant but suggests that IE is an acceptable adjuvant regimen in patients with poor necrosis rates.

\section{Conclusions}

Adult and young adult osteosarcoma patients under 40 years with localised osteosarcoma treated at our institution have equivalent outcomes compared to paediatric patients. Patients over 40 years are more challenging and are more likely to experience toxicity. Switching chemotherapy to ifosfamide/etoposide alone in patients with poor necrosis rates post neoadjuvant methotrexate, doxorubicin, cisplatin is a feasible regimen, but where these drugs fit in the treatment paradigm remains to be established.

\section{Additional files}

Additional file 1: Table S1. Chemotherapy protocol for resectable disease.

Additional file 2: Table S2. Patient characteristics.

Additional file 3: Table S3. Multivariate Cox-regression analysis of prognostic factors for event free survival and overall survival.

\section{Authors' contributions}

KC participated in data collection, study design and data interpretation. EW participated in data collection, study design and data interpretation. RE participated in data collection and analysis. PD participated in study design. COK participated in study design and manuscript revision. $\mathrm{BH}$ participated in study design. GOT participated in study design and manuscript revision. SD participated in study design. EK participated in study design and data interpretation. SE participated in study design and data interpretation. DC participated in study design, data collection and interpretation and manuscript revision. All authors read and approved the final manuscript. 


\section{Author details}

${ }^{1}$ Department of Medical Oncology, Mater Misericordiae University Hospital, Eccles Street, Dublin 7, Ireland. ${ }^{2}$ Gynaecologic Medical Oncology Service, Memorial Sloan-Kettering Cancer Centre, 300 East 66th Street, New York, NY 10065, USA. ${ }^{3}$ Department of Histopathology, Mater Misericordiae University Hospital, Eccles Street, Dublin 7, Ireland. ${ }^{4}$ Department of Orthopaedic Surgery, Cappagh National Orthopaedic Hospital, Finglas, Dublin 11, Ireland. ${ }^{5}$ Department of Radiology, Mater Misericordiae University Hospital, Eccles Street, Dublin 7, Ireland.

\section{Compliance with ethical guidelines}

\section{Competing interests}

The authors declare that they have no competing interests.

Received: 2 May 2015 Accepted: 30 June 2015

Published online: 14 July 2015

\section{References}

1. Bielack S, Carrle D, Casali PG, Group EGW (2009) Osteosarcoma: ESMO clinical recommendations for diagnosis, treatment and follow-up. Ann Oncol 20(Suppl 4):137-139

2. Huvos AG (1986) Osteogenic sarcoma of bones and soft tissues in older persons. A clinicopathologic analysis of 117 patients older than 60 years. Cancer 57(7):1442-1449

3. Ottaviani G, Jaffe N (2009) The epidemiology of osteosarcoma. Cancer Treat Res 152:3-13

4. Bielack SS, Kempf-Bielack B, Delling G, Exner GU, Flege S, Helmke K et al (2002) Prognostic factors in high-grade osteosarcoma of the extremities or trunk: an analysis of 1,702 patients treated on neoadjuvant cooperative osteosarcoma study group protocols. J Clin Oncol Off J Am Soc Clin Oncol 20(3):776-790

5. Marcove RC, Miké V, Hajek JV, Levin AG, Hutter RV (1970) Osteogenic sarcoma under the age of twenty-one. A review of one hundred and forty-five operative cases. J Bone Joint Surg Am 52(3):411-423

6. Goorin AM, Schwartzentruber DJ, Devidas M, Gebhardt MC, Ayala AG, Harris MB et al (2003) Presurgical chemotherapy compared with immediate surgery and adjuvant chemotherapy for nonmetastatic osteosarcoma: Pediatric Oncology Group Study POG-8651. J Clin Oncol 21(8):1574-1580

7. Provisor AJ, Ettinger LJ, Nachman JB, Krailo MD, Makley JT, Yunis EJ et al (1997) Treatment of nonmetastatic osteosarcoma of the extremity with preoperative and postoperative chemotherapy: a report from the Children's Cancer Group. J Clin Oncol Off J Am Soc Clin Oncol 15(1):76-84

8. Hogendoorn PC, Athanasou N, Bielack S, De Alava E, Dei Tos AP, Ferrari S et al (2010) Bone sarcomas: ESMO clinical practice guidelines for diagnosis, treatment and follow-up. Ann Oncol 21(Suppl 5):v204-v213

9. Rosen $G$ (1985) Preoperative (neoadjuvant) chemotherapy for osteogenic sarcoma: a 10 year experience. Orthopedics 8(5):659-664

10. Ta HT, Dass CR, Choong PF, Dunstan DE (2009) Osteosarcoma treatment: state of the art. Cancer Metastasis Rev 28(1-2):247-263

11. Rosen G, Marcove RC, Huvos AG, Caparros BI, Lane JM, Nirenberg A et al (1983) Primary osteogenic sarcoma: eight-year experience with adjuvant chemotherapy. J Cancer Res Clin Oncol 106(Suppl):55-67

12. Bacci G, Bertoni F, Longhi A, Ferrari S, Forni C, Biagini R et al (2003) Neoadjuvant chemotherapy for high-grade central osteosarcoma of the extremity. Histologic response to preoperative chemotherapy correlates with histologic subtype of the tumor. Cancer 97(12):3068-3075

13. Hauben El, Weeden S, Pringle J, Van Marck EA, Hogendoorn PC (2002) Does the histological subtype of high-grade central osteosarcoma influence the response to treatment with chemotherapy and does it affect overall survival? A study on 570 patients of two consecutive trials of the European Osteosarcoma Intergroup. Eur J Cancer 38(9):1218-1225

14. Bacci G, Longhi A, Versari M, Mercuri M, Briccoli A, Picci P (2006) Prognostic factors for osteosarcoma of the extremity treated with neoadjuvant chemotherapy: 15-year experience in 789 patients treated at a single institution. Cancer 106(5):1154-1161
15. Smeland S, Müller C, Alvegard TA, Wiklund T, Wiebe T, Björk O et al (2003) Scandinavian Sarcoma Group Osteosarcoma Study SSG VIII: prognostic factors for outcome and the role of replacement salvage chemotherapy for poor histological responders. Eur J Cancer 39(4):488-494

16. Anninga JK, Gelderblom H, Fiocco M, Kroep JR, Taminiau AH, Hogendoorn PC et al (2011) Chemotherapeutic adjuvant treatment for osteosarcoma: where do we stand? Eur J Cancer 47(16):2431-2445

17. Goorin AM, Harris MB, Bernstein M, Ferguson W, Devidas M, Siegal GP et al (2002) Phase II/III trial of etoposide and high-dose ifosfamide in newly diagnosed metastatic osteosarcoma: a pediatric oncology group trial. J Clin Oncol Off J Am Soc Clin Oncol 20(2):426-433

18. Schwartz CL, Wexler LH, Devidas M, Goorin A, Grier H, Meyers P et al (2004) P9754 therapeutic intensification in non-metastatic osteosarcoma: a COG trial. In J Clin Oncol (meeting abstracts) July 2004, vol. 22, no. 14_suppl8514

19. Marina N, Smeland S, Bielack SS, Bernstein M, Jovic G, Hook JM et al (2014) MAPIE vs MAP as postoperative chemotherapy in patients with a poor response to preoperative chemotherapy for newly-diagnosed osteosarcoma: results from EURAMOS-1 (Paper 032). Presented at Connective Tissue Oncology Society (CTOS) 2014.

20. Simon MA, Aschliman MA, Thomas N, Mankin HJ (1986) Limb-salvage treatment versus amputation for osteosarcoma of the distal end of the femur. J Bone Joint Surg Am 68(9):1331-1337

21. Rougraff BT, Simon MA, Kneisl JS, Greenberg DB, Mankin HJ (1994) Limb salvage compared with amputation for osteosarcoma of the distal end of the femur. A long-term oncological, functional, and quality-of-life study. J Bone Joint Surg Am 76(5):649-656

22. Ries LA, Smith M, Gurney JG et al (eds) (1999) Cancer incidence and survival among children and adolescents: United States SEER Program 1975-1995

23. Mirabello L, Troisi RJ, Savage SA (2009) International osteosarcoma incidence patterns in children and adolescents, middle ages and elderly persons. Int J Cancer 125(1):229-234

24. Carsi B, Rock MG (2002) Primary osteosarcoma in adults older than 40 years. Clin Orthop Relat Res 397:53-61

25. Grimer RJ, Cannon SR, Taminiau AM, Bielack S, Kempf-Bielack B, Windhager R et al (2003) Osteosarcoma over the age of forty. Eur J Cancer 39(2):157-163

26. Longhi A, Errani C, Gonzales-Arabio D, Ferrari C, Mercuri M (2008) Osteosarcoma in patients older than 65 years. J Clin Oncol Off J Am Soc Clin Oncol 26(33):5368-5373

27. Harting MT, Lally KP, Andrassy RJ, Vaporciyan AA, Cox CS, Hayes-Jordan A et al (2010) Age as a prognostic factor for patients with osteosarcoma: an analysis of 438 patients. J Cancer Res Clin Oncol 136(4):561-570

28. Bacci G, Ferrari S, Donati D, Longhi A, Bertoni F, Di Fiore M et al (1998) Neoadjuvant chemotherapy for osteosarcoma of the extremity in patients in the fourth and fifth decade of life. Oncol Rep 5(5):1259-1263

29. Kager L, Zoubek A, Pötschger U, Kastner U, Flege S, Kempf-Bielack B et al (2003) Primary metastatic osteosarcoma: presentation and outcome of patients treated on neoadjuvant Cooperative Osteosarcoma Study Group protocols. J Clin Oncol Off J Am Soc Clin Oncol 21(10):2011-2018

30. Aljubran AH, Griffin A, Pintilie M, Blackstein M (2009) Osteosarcoma in adolescents and adults: survival analysis with and without lung metastases. Ann Oncol 20(6):1136-1141

31. Bielack SS, Kempf-Bielack B, Branscheid D, Carrle D, Friedel G, Helmke Ket al (2009) Second and subsequent recurrences of osteosarcoma: presentation, treatment, and outcomes of 249 consecutive cooperative osteosarcoma study group patients. J Clin Oncol Off J Am Soc Clin Oncol 27(4):557-565

32. Chou AJ, Merola PR, Wexler LH, Gorlick RG, Vyas YM, Healey JH et al (2005) Treatment of osteosarcoma at first recurrence after contemporary therapy: the Memorial Sloan-Kettering Cancer Center experience. Cancer 104(10):2214-2221

33. Bacci G, Briccoli A, Longhi A, Ferrari S, Mercuri M, Faggioli F et al (2005) Treatment and outcome of recurrent osteosarcoma: experience at Rizzoli in 235 patients initially treated with neoadjuvant chemotherapy. Acta Oncol 44(7):748-755

34. Bielack SS, Kempf-Bielack B, Winkler K (1996) Osteosarcoma: relationship of response to preoperative chemotherapy and type of surgery to local recurrence. J Clin Oncol Off J Am Soc Clin Oncol 14(2):683-684 
35. Lewis IJ, Nooij MA, Whelan J, Sydes MR, Grimer R, Hogendoorn PC et al (2007) Improvement in histologic response but not survival in osteosarcoma patients treated with intensified chemotherapy: a randomized phase III trial of the European Osteosarcoma Intergroup. J Natl Cancer Inst 99(2):112-128

36. Jaffe N, Gorlick R (2008) High-dose methotrexate in osteosarcoma: let the questions surcease - time for final acceptance. J Clin Oncol Off J Am Soc Clin Oncol 26(27):4365-4366

37. Marina N, Bielack S, Whelan J, Smeland S, Krailo M, Sydes MR et al (2009) International collaboration is feasible in trials for rare conditions: the EURAMOS experience. Cancer Treat Res 152:339-353

38. Bielack SS, Smeland S, Whelan JS, Marina N, Jovic G, Hook JM et al (2015) Methotrexate, doxorubicin, and cisplatin (map) plus maintenance pegylated interferon Alfa-2b versus MAP alone in patients with resectable high-grade osteosarcoma and good histologic response to preoperative MAP: first results of the EURAMOS-1 good response randomized controlled trial. J Clin Oncol. doi:10.1200/JCO.2014.60.0734
39. Carli M, Passone E, Perilongo G, Bisogno G (2003) Ifosfamide in pediatric solid tumors. Oncology 65(Suppl 2):99-104

40. Ferrari S, Ruggieri P, Cefalo G, Tamburini A, Capanna R, Fagioli F et al (2012) Neoadjuvant chemotherapy with methotrexate, cisplatin, and doxorubicin with or without ifosfamide in nonmetastatic osteosarcoma of the extremity: an Italian sarcoma group trial ISG/OS-1. J Clin Oncol Off J Am Soc Clin Oncol 30(17):2112-2118

41. Bacci G, Briccoli A, Ferrari S, Longhi A, Mercuri M, Capanna R et al (2001) Neoadjuvant chemotherapy for osteosarcoma of the extremity: longterm results of the Rizzoli's 4th protocol. Eur J Cancer 37(16):2030-2039

42. Bruland $\varnothing$, Bauer $H$, Alvegaard T, Smeland S (2009) Treatment of osteosarcoma. The Scandinavian Sarcoma Group experience. Cancer Treat Res 152:309-318

\section{Submit your next manuscript to BioMed Central} and take full advantage of:

- Convenient online submission

- Thorough peer review

- No space constraints or color figure charges

- Immediate publication on acceptance

- Inclusion in PubMed, CAS, Scopus and Google Scholar

- Research which is freely available for redistribution

Submit your manuscript at

www.biomedcentral.com/submit

(O) Biomed Central 